\title{
BMJ Open A modified Delphi study towards developing a guideline to inform policy on fetal alcohol spectrum disorders in South Africa: a study protocol
}

\author{
Babatope O Adebiyi, ${ }^{1}$ Ferdinand C Mukumbang, ${ }^{1}$ Kufre J Okop, ${ }^{1}$ \\ Anna-Marie Beytell ${ }^{2}$
}

To cite: Adebiyi BO,

Mukumbang FC, Okop KJ, et al. A modified Delphi study towards developing a guideline to inform policy on fetal alcohol spectrum disorders in South Africa: a study protocol. BMJ Open 2018;8:e019907. doi:10.1136/ bmjopen-2017-019907

- Prepublication history for this paper is available online. To view these files, please visit the journal online (http://dx.doi. org/10.1136/bmjopen-2017019907).

Received 3 October 2017 Revised 6 March 2018 Accepted 20 March 2018
Check for updates

${ }^{1}$ School of Public Health, University of the Western Cape, Cape Town, South Africa

${ }^{2}$ Department of Social Work, University of the Western Cape, Cape Town, South Africa

Correspondence to Mr Babatope 0 Adebiyi; atommega@yahoo.com

\section{ABSTRACT}

Introduction Maternal alcohol consumption during pregnancy can result in mental and physical birth defects in individuals. These birth defects are usually described as fetal alcohol spectrum disorders (FASDs). With an estimated 183-259 per 1000 children born with FASDs, South Africa is identified to have the highest prevalence of FASDs in the world. Nevertheless, there is a lack of appropriate policies, guidelines and interventions addressing the issues around FASDs. This protocol outlines a proposed process for developing a guideline to inform policies on FASDs.

Methods and analysis This process will have three phases. Phase I will be carried out in three steps; we plan to conduct a document review of available policies on the prevention and management of FASDs and update the existing systematic review on FASDs interventions. The aim of the two reviews is to explore the availability and content of existing policies and global interventions on FASDs. In addition, we will conduct two exploratory qualitative studies to obtain the perspectives of various stakeholders on the existing or possible guidelines and policies for the management of FASDs and available interventions and services. In phase II, we will aggregate the findings of the previous phase to develop a prototype guideline. In phase III, using the developed prototype, we will apply the Delphi approach with experts on FASDs, soliciting their opinions on the nature and content of the proposed guidelines for policies. The information gathered will be used to modify the prototype to formulate a policy guideline on FASDs. The data will be analysed using thematic analysis and narrative synthesis.

Ethics and dissemination Ethical clearance has been obtained from the ethics committee of the university and governmental departments. The findings will be disseminated through publications and the guideline will be submitted to relevant departments.

\section{INTRODUCTION}

Fetal alcohol spectrum disorders (FASDs) refer to an array of birth disorders related to fetal exposure to alcohol during pregnancy. FASDs are classified under four broad groups: fetal alcohol syndrome (FAS), partial FAS,
Strengths and limitations of this study

- The study proposes the use of multiple sources for data collection towards developing a guideline to inform policy on fetal alcohol spectrum disorders (FASDs).

- It also proposes a proven methodology - the Delphi technique to develop the guideline.

- A potential limitation of this study is that it will not include individuals with FASDs.

- This study is expected to inform policy on FASDs nationally; however, it will be conducted in only one province out of nine provinces of South Africa.

alcohol-related neurodevelopment disorders (ARNDs) and alcohol-related birth defects. ${ }^{1}$ Of these four groups, FAS is identified as having the most serious anomalies. ${ }^{23}$

FASDs may lead to primary and secondary disabilities. ${ }^{4}$ Primary disabilities are those that the child is born with, ${ }^{5}$ usually associated with damages to the brain domains that are responsible for physical motor skills, sensory processing skills, cognition, communication skills, academic achievement, memory skills, executive functioning and abstract reasoning, attention and adaptive skills. ${ }^{6} 7$ The brain damages could also result in physical abnormalities such as antenatal and postnatal growth retardation, eye and ear malformations, mouth and jaw deformation, skeletal defects, organ pathology, sensory deficits and impaired immune system. ${ }^{1}$

Secondary disabilities relate to those disabilities that develop because of untimely and inappropriate interventions to primary disabilities-consequences of unaddressed primary disabilities. These include fatigue, frustration, anxiety, fearfulness, rigid, resistant, argumentative behaviour, becoming overwhelmed, shut down (withdrawn), a poor self-concept, feelings of failure, low 
self-esteem, isolation, acting out, aggression, family and/ or school problems, depression and other mental health problems, trouble with the law, drug and alcohol problems, problems with employment and homelessness.

A systematic review conducted to identify maternal risk factors for FASDs showed that the maternal demographic (socioeconomic status, employment status, educational status, marital status, religion, living area, income and age) and psychiatric-including neuropsychological (psychiatric comorbidity, identified stress, physical aggression and sexual abuse)—factors play significant roles. ${ }^{9}$ The review also showed that family, social (family lifestyle, drinking habit, alcohol use, illegal drugs use and tobacco use) and pattern of alcohol consumption could predispose women to have babies with FASDs. ${ }^{9}$

In South Africa, the national prevalence of FASD ranges from 29 to 290 per 1000 live births. ${ }^{10}$ Some provinces such as the Northern and Western Cape of South Africa have particularly registered high FASD prevalence. In the Northern Cape, an estimated 88 per 1000 of firstgrade pupils were reported to have FASDs in $2008 .{ }^{11}$ In 2015, although the prevalence had dropped to 63.9 per 1000 , the prevalence was still relatively high. ${ }^{12}$ In the Western Cape, the prevalence of FASDs among primary school pupils was estimated at 89.2 per 1000 in $2007 .{ }^{13}$ By 2013, the prevalence of FASDs among first-grade pupils had doubled (135.1 to 207.5 per 1000), ${ }^{14}$ and another increase (170 to 233 per 1000) was recorded in $2015 .{ }^{15}$

There is evidence that strategies designed to prevent FASDs and to care and support persons affected by FASDs are not effectively implemented, particularly in low/ middle-income countries. ${ }^{16}$ Peadon $e t ~ a l^{17}$ and Reid et $a l^{18}$ also reported the lack of good-quality studies and limited strong evidence for specific interventions in managing FASDs. The authors, therefore, advocated for interventions targeting the specific clinical and neuropsychological deficits usually seen in individuals with FASDs.

\section{BACKGROUND}

South Africa is reportedly having escalating levels of alcohol consumption, ${ }^{19}$ particularly in the informal settlements of the Northern and Western Cape provinces. ${ }^{20-23}$ The prevalence of alcohol use among pregnant women in the Cape Metropole of the Western Cape province of South Africa is estimated at $19.6 \%$, and in poor communities of the Western and Northern Cape provinces of South Africa, FASDs are endemic. ${ }^{1324}$ Maternal alcohol drinking during pregnancy, especially in rural and informal settlements, has led FASDs become an increasing concern in South Africa.

Generally, FASDs are relatively unknown among health professionals and have not been given adequate consideration in policies, programmes and interventions. ${ }^{25}$ This is probably because FASDs remain unformalised medical diagnoses since they were first identified and described 40 years earlier. ${ }^{25}$ Despite the apparent lack of interest in FASDs, efforts towards developing guidelines for the diagnoses $^{2627}$ and management ${ }^{28}$ of FASDs have been made, especially towards recognising FASD as a public health problem. Because FASDs are preventable, there have also been advocacy efforts for the implementation of various prevention programmes. These advocacy strategies call for the availability of adequately resourced alcohol control services, support for pregnant women and a sustainable commitment from communities, service providers and the government in addressing alcohol-related health and psychological problems. ${ }^{29} 30$ Nevertheless, for these strategies to be effective and sustainable, a governing policy and coordinated efforts are required.

Globally, there are inadequate policy and guidelines for the management and prevention of FASDs. A review on FASDs in Africa reported gaps in policy and service implementation. ${ }^{31}$ In South Africa, only two of the national policy documents, namely, the National Human Genetics Policy Guidelines for the Management and Prevention of Genetic Disorders, Birth Defects and Disabilities (2001) and the National Drug Master Plan (2007) mentioned the term FAS, and none mentioned FASD. ${ }^{32}$ In addition, the South African Guidelines for Maternity Care (2002) only suggests that maternal alcohol, tobacco and other substances use behaviours should be taken into consideration when taking a medical history of a pregnant woman. ${ }^{32}$ The limited occurrence of issues related to FASDs in policy documents offers a reflection on the extent to which FASDs are (not) being considered in policy.

A current national hospital-based birth defects surveillance system developed by the National Department of Health also excludes some FASDs. ${ }^{32}$ This surveillance system mainly considers birth defects that can be clinically diagnosed at birth or few days after birth, which can only be identified during postnatal hospitalisation. The exclusion of some FASDs in the birth defects surveillance system is because of the difficulty of diagnosing certain FASDs at birth as most of their features are rarely manifested at birth. In addition, it requires a multidisciplinary team to arrive at a definitive diagnosis of FASDs. ${ }^{32}$ The above gaps speak to the need to develop relevant policies and guidelines to identify FASDs and to guide strategic implementation of appropriate interventions to address FASDs in South Africa.

In 2001, the Western Cape provincial executive council acknowledged FASDs as a provincial health priority for the years 2001 and 2002. ${ }^{33}{ }^{34}$ This led to the establishment of the Western Cape provincial FAS reference and working group (now called FASD Task Team). The FASD Task Team of South Africa comprises members from the Department of Health, the Department of Social Services, the Department of Education, the Medical Research Council, the University of Stellenbosch, the University of Cape Town and non-profit organisations (NPOs) (Foundation for Alcohol Related Research, Dopstop and Pebbles Project). The task team is headed by the Provincial Maternal, Child and Women's Health deputy director. ${ }^{334}$ The aims of the FASD Task Team is to raise awareness on FASDs, create 
strategies for the prevention of FASDs and share skills and information. ${ }^{33} 34$

The FASD Task Team has been successful in developing FASDs training manuals for healthcare workers, educators, school psychologists, social workers and other professionals working with individuals with FASDs, their families and caregivers. In addition, the team designed posters for creating awareness on FASDs, organising special events to improve public awareness on international FASDs days, organising FASDs training workshops and developing a website and Facebook page on FASD. ${ }^{33} 34$

The above development is an important and commendable step in developing the capacity to support the prevention and management of FASDs at the provincial level. Nevertheless, a coordinated effort to facilitate the prevention and management of FASDs at the national level is required. To this end, we are proposing a national FASDs guideline that will inform policy on coordinated and multisectoral response to FASDs in South Africa. In this protocol, we propose a systematic approach towards developing a guideline to inform policy for the prevention and management of FASDs in South Africa.

\section{RESEARCH OBJECTIVES}

To develop a guideline for the prevention and management of FASDs in South Africa, we aimed to achieve the following objectives:

- To review policies and their contents on FASDs in South Africa.

- To explore and describe the content of FASDs policies from the perspective of policy-makers.

- To provide an update on the interventions on FASDs internationally and in South Africa.

- To explore and describe the perspectives of service providers involved in the implementation of identified FASDs intervention activities.

- To apply the Delphi technique to guide the development of guidelines for an integrated policy on FASDs for the South African context.

\section{Understanding policy guidelines}

According to the WHO, a guideline is a document that contains recommendations for clinical or public health interventions. ${ }^{35} 36$ It is also described as a document that makes evidence-based recommendations for averting and treating certain conditions, improving health, managing medicines, planning of services for the community, development of an intervention for enhancing population health and delivering social care for people. ${ }^{37}$ Thus, guidelines promote individualised and integrated care, help service providers and recipients and other stakeholders to make informed decisions and serve as a response to a condition that needs urgent attention. ${ }^{35-38}$ Therefore, a guideline is considered as a set of systematically developed statements, recommendation or best practice to give a framework for policy implementation. ${ }^{38}$ As applies to this protocol, we considered a guideline as a document that contains recommendations that will inform the development of integrated policy on FASDs.

\section{Adopted approach}

In developing the guideline for FASDs, we adopted the WHO's approach (steps) as stipulated in the WHO's Handbook for guideline development. ${ }^{35} 36$

1. We agreed to design a guideline for the prevention and management of FASDs based on informal discussions with relevant stakeholders on the need for a policy governing the prevention and management efforts of FASDs.

2. We planned on scoping of the literature and conducting a needs assessment.

3. After gathering preliminary information, the team formulated key PICO questions ${ }^{39}$ :

A. Population-individuals with FASDs.

B. Intervention-a guideline to inform policy.

C. Comparison-(not applicable).

D. Outcomes-a guideline for the prevention and management of FASDs.

4. After agreeing on the need to design the guideline to inform policy on the prevention and management of FASDs, the next step involved is designing the project protocol (which is reported in this paper).

5. The team applied for ethics clearance for the project from the University of the Western Cape-this has been obtained.

6. The next step will entail conducting the various studies and exercises outlined in this protocol.

7. The team will develop and prepare the guideline to inform policy on FASDs based on the findings of the various studies and the outcomes of the various exercises.

8. The team will disseminate the developed guideline through various channels including having feedback meetings with the various stakeholders and sharing our findings, publish the guideline in a peer-reviewed journal, present the findings in a journal club meeting organised at the provincial Department of Health and at other national and international conferences. ${ }^{35} 36$

Some of the steps outlined in the WHO's handbook were adapted to our context. The reason for the adaptation is that some parts of the process are not applicable to this study and the other parts are beyond the scope of this project.

\section{Proposed methodology}

The development of the guideline will follow three phases: (I) information gleaning, (II) development of prototype and (III) Delphi techniques and developing of guidelines (figure 1). The importance of involving proven methods, which are both inclusive and participatory, was considered as essential for developing a socially acceptable FASDs guideline for South Africa.

A proposed methodological approach for the development of guidelines for FASDs policy. 
PHASE I: INFORMATION GLEANING

Exploratory approaches will be employed to gather relevant information for the development of a prototype FASD guidelines

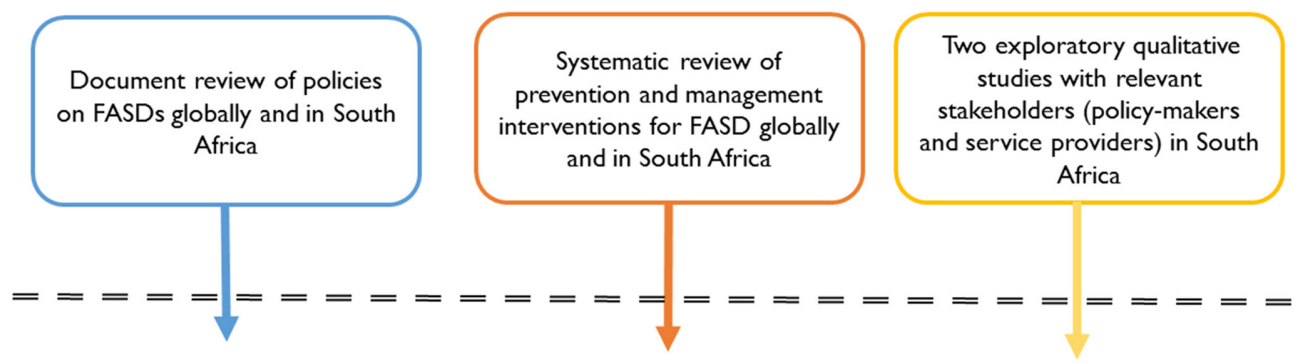

PHASE I I:PROTOTYPE DEVELOPMENT

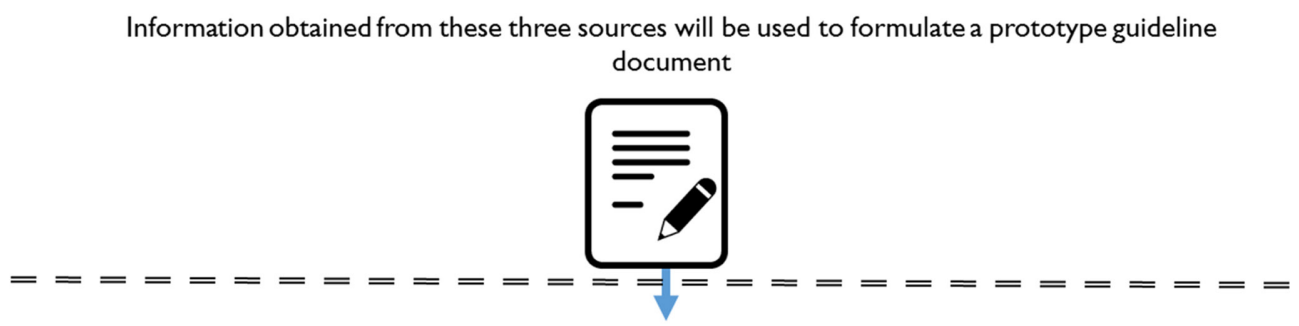

PHASE III:REFINING THE PROTOTYPE

The process of fine-tuning and refining the prototype guideline will involve repeating consultations with some key stakeholders in the Delphi approach

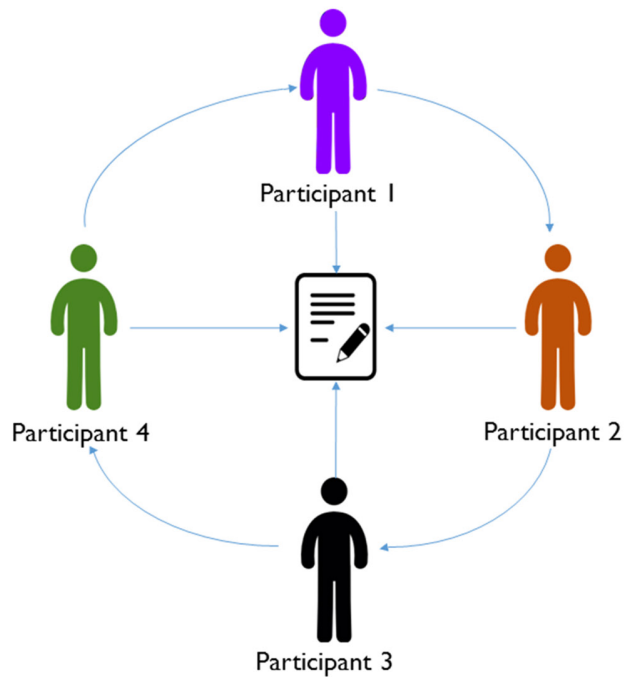

The outcome of the Delphi process is to obtained a more refined guideline that can inform policies for the prevention and management of FASDs

Figure 1 A proposed methodological approach for the development of guidelines for fetal alcohol spectrum disorders (FASDs) policy.

\section{Phase I: information gleaning}

In phase I, three steps will be undertaken with the purpose of obtaining diverse information to develop a prototype guideline. To this end, we plan to conduct a document review on FASDs policies and a systematic review on FASDs interventions. The document review will consider policies developed South Africa and the systematic review will focus on interventions globally with a focus on South Africa. In addition, we will identify relevant policy-makers and service providers from the departments of health, education and social development and engage with them through in-depth interviews and focus group discussions to obtain information on existing policies and interventions on FASDs in South Africa. This information-gleaning phase will be conducted in the following steps.

Step 1: document review of policy on FASD in South Africa The researchers will review available relevant documents such as policy documents and guidelines on FASDs. The aim of this review is to explore the availability and content 
of existing guidelines and policies on FASDs. Websites (government and NPOs) and databases will be searched for these documents. All the policies or guidelines that focused on any aspect of FASDs will be included. A narrative analysis will be used to analyse the relevant documents identified. The information that will be obtained will inform the development of a prototype guideline. Following the document review, a systematic review will be undertaken.

\section{Step 2: systematic review of interventions for FASDs globally and} in South Africa

The purpose of the systematic review will be to update the current systematic review on FASDs' 'Fetal alcohol spectrum disorder interventions across the life span ${ }^{, 18}$ to include interventions for prevention. The aim of the systematic review will be to systematically locate, appraise and synthesise intervention on FASDs. The systematic review will consider the following review question: 'What are the prevention and management interventions that are available on FASDs globally and in South Africa?' In conducting this review, the researchers will follow nine steps of a systematic review. ${ }^{40}$ These steps include formulating a review question, defining search strategy, establishing inclusion criteria and exclusion criteria, choosing a method for the review, conducting methodological quality and critical appraisal, extracting data, analysing and synthesising data and writing a report.

What are the interventions that are available on FASDs globally and in South Africa? To answer this question, databases will be searched for articles discussing or evaluating interventions for the prevention and management of FASDs. These databases include: Ebsco Host (Academic Search Complete, ERIC, SoINDEX, Health Source: Nursing/Academic Edition, Cumulative Index of Nursing and Allied Health and PsycARTICLES), Science Direct, Springer Link, Wiley Online Library, JSTOR and SAGE journals, PubMed, Cochrane Library, Sabinet and NEXUS will be searched and retrieved for the period covering 2007 to 2017 using intervention and FASDs as search terms. Furthermore, the references of the retrieved article will be used to search for more articles.

The titles and abstracts of the articles will be screened using inclusion/exclusion criteria, and duplicates will be removed. After screening, the articles that meet the inclusion criteria will be read and their methodological quality evaluated. In addition, the included articles will be appraised using a critical appraisal sheet. A narrative synthesis will be used for data analysis. A narrative synthesis can be described as a descriptive written summary of included studies and their findings. ${ }^{41}$ The data extraction tool will consist of four distinct sections, namely the general description, a methodological appraisal, the content intervention and the analysis of the results. While conducting and reporting the reviews, we will follow the guidelines for systematic reviews.
Step 3: exploratory qualitative research

Two exploratory qualitative studies will be conducted. The first qualitative study will explore the availability of guidelines/policies for the prevention and management of FASDs within the South African health system and the need to develop a policy for the prevention and management of FASDs. This study will target the policy-makers (administrators involved in policy formulation and programme monitoring on FASDs and related disabilities) from three relevant departments: Department of Education, Department of Health and Department of Social Development.

The second qualitative study will be aimed at identifying existing interventions and guideline/policy statements that are being used by the service providers (individuals involved in providing services and interventions on FASDs and related disabilities) for the prevention and management of FASDs. Relevant service providers include paediatricians, nurses, social workers and occupational therapist. Policy-makers and service providers from the Department of Education, Department of Health and Department of Social Development will be interviewed as part of the processes of developing guidelines that will inform policy on FASDs. These groups of individuals are deemed knowledgeable to provide relevant information towards developing FASDs guidelines.

Purposive sampling will be employed in selecting participants. For the policy-makers, we will include those individuals with about 5 years of experience in policy on FASDs or related disabilities. Similarly, service providers working in primary healthcare, hospital, schools, social development and NPOs with at least 5 years of experience in providing interventions and services to individuals with FASDs will be potential participants. The researchers intend to sample three policy-makers from each department and conduct three focus group discussions in each department with an average of six to eight participants. Data saturation will be used to determine the sample sizes in the two qualitative studies.

In the first qualitative study, data will be collected through in-depth interviews with policy-makers using an interview guide. Open-ended questions will be used to start the interviews and follow-up questions will be used to probe for additional explanations when required. The study participants will be asked various questions on available policies on FASDs, the coordination of FASDs interventions and relevant aspects of FASDs to inform the FASDs guidelines. Each interview will last for about 30-60 min and each interview will be audio-recorded with the permission from the participants.

Data, in the second qualitative study, will be collected through focus group discussions with selected service providers using a discussion schedule to explore available interventions, services and policies in practice. Focus groups use unstructured discussion subjects involving small ranges of subjects (6-10). It is cost-effective and time efficient and participants are allowed to express their viewpoints in detail. Discussants are often motivated 
to contribute more in the presence of their coparticipants. ${ }^{42}$ In addition, focus group discussions can give rich information as discussants contribute in moderated discussions. An audio recorder will be used to capture information from the discussants. Notes will also be taken during the interview, and the interview recordings will be transcribed for data analysis.

The data will be analysed using thematic content analysis technique. ${ }^{43}$ Both inductive-making interpretations of the raw data-and deductive-using a frameworkanalytic approaches will be used to analyse the data. ${ }^{44}$ The framework will be developed from the prevention and management aspects of FASDs. The researchers will use Creswell's data analysis spiral. ${ }^{45}$ This process involves reading and memoing, then describing, classifying and interpreting the data according to themes and categories and finally visualising and representing the themes and categories. An independent coder will also be used to ensure trustworthiness.

The rigour in the qualitative studies, which is trustworthiness will be established through credibility, transferability, dependability, conformability and reflexivity. ${ }^{46}$ In addition, the reporting of the qualitative findings will follow the consolidated criteria for reporting qualitative research. $^{47}$

\section{Phase II: development of prototype}

In the second phase, information obtained from the document review, systematic review and in-depth and focus group interviews will be conflated to develop a prototype guideline for a FASDs policy. The researchers will engage and agree on consensus interpretations of the findings to develop the prototype guideline. We selected this approach because it has been successfully applied in the guideline development process by other researchers in developing guidelines for various issues. ${ }^{4-50}$

\section{Phase III: Delphi techniques and developing of guidelines}

In the third phase, we will apply the Delphi approach to refine the developed prototype. The use of the Delphi approach is recommended by the WHO Handbook for guideline development. ${ }^{35} 36$ The Delphi technique is a structured communication technique initially developed as a systematic, interactive forecasting method, which relies on a panel of experts. ${ }^{51}$ It is common for experts to answer questionnaires in two or more rounds. ${ }^{52}$ The researcher gives an anonymous summary of the experts' predictions from the previous round and the reasons they provided for their judgements. Experts are encouraged to revise their earlier responses in the light of the replies of other members in the forum. This process narrows the wide range of answers and converges the group answers towards an agreed answer. ${ }^{53}$ The process is stopped after a predefined stop criterion and the mean or median scores of the final rounds determine the results. ${ }^{51}$

There is no agreement on the sample size of participants in the Delphi discussion forum. We will identify relevant policy-makers, health service providers and health policy advocates to participate in a Delphi contribution forum to refine the prototype. Some of the Delphi participants will include those who participated in any of the two qualitative studies. This is because experts on FASDs issues are few, and it may be challenging to get a complete set of different participants.

We will start by identifying the experts and contacting them to explain the research. We will then purposefully select 30 experts using the following criteria: top management decision-makers; policy-makers; FASDs service providers and FASDs researchers with about 5 years of experience relevant to FASDs policies, services and intervention or research. ${ }^{54}$ These experts will be contacted via email and telephone for their contributions.

The developed prototype will be used to engage in a Delphi technique with experts on FASDs to develop the proposed guidelines. Descriptions of prevention and management interventions identified in phase I will be used to design Likert statements to evaluate the perceptions of the experts on prevention modalities, screening components and management methods. We will solicit their opinions on what should be included or excluded in the proposed guidelines for policies. Participants will be asked to rate their agreement with each statement on a five-point Likert scale, which will range from 'strongly agree' to 'strongly disagree', and a response option of 'no comment' will be provided to enable participants to indicate that a statement was outside their area of expertise. ${ }^{55}$

The data generated will be analysed, and these will be used to modify the prototype, which will be used as an instrument for the second round. The experts will be asked to review the items summarised by the researchers based on the information provided in round 1 and they will be asked to rate or rank-order items to establish priorities among items and to reach a consensus. The information generated in the second round will then be analysed to refine the prototype to obtain the guidelines. The process of guideline development is expected to be evolving, thus we plan to follow a thorough process and procedures as resource and time would permit.

\section{Patient and public involvement}

Patient and public will not be involved in the study. The findings of the study will be disseminated to the study participants through emails.

\section{DISCUSSION}

The essence of the proposed guideline is to inform policy development on FASDs. While having informal discussions with the head of a non-governmental organisation, a leading organisation in conducting prevalence studies and rolling out prevention programme on FASDs, we understood that focused guidelines and policies to facilitate effective interventions on FASDs are non-existent. The administrators of the departments of Education, Health and Social Development in the Western Cape province also revealed that drinking alcohol remains a major 
concern, and FASDs are among the negative outcomes of irresponsible drinking practices.

These administrators pointed out that various efforts have been made, ranging from awareness programmes to funding of NGOs to response to FASDs, but these efforts are not being coordinated because there are no specific guidelines or policies on FASDs. Service providers consulted also raised similar concerns. Nevertheless, the service providers suggested that generic programmes are in place to provide health education on alcohol use during pregnancy to pregnant women and that they would sometimes refer suspected cases of FASDs for proper diagnosis. Of course, these practices are not specifically targeting the problem of FASDs or addressing the issues around FASDs in an intended manner. The lack of focused attention accorded to FASDs prompted the conception to develop guidelines that will enhance the prevention and management of FASDs.

The strength of using the approach outlined in this protocol lies in the fact that it has been successfully used to develop various guidelines for interventions to combat diseases and improve services. In addition, the recommendations from the guidelines will be rooted in a comprehensive and objective assessment of the available evidence and a clear pathway on how recommendations are generated. The demerit of this process is that it requires a lot of resources, time and expertise. The limitation of this study might be that the researchers will not include the individuals with FASDs and their families directly in the study. Another limitation is that the study is restricted to the Western Cape region of South Africa, which might affect the generalisability of the study findings.

It has been reported that there are no guidelines for determining consensus, sample size and sampling techniques when using the Delphi approach. This is because these aspects of the Delphi technique require a lot of time and participants' commitment; consequently, a dropout is likely to happen, which leads to a low response rate and a delay in data analysis between rounds. ${ }^{5657}$ To mitigate the impact of these potential challenges, the researchers have decided to adapt the sample size to a manageable number to favour the in-depth engagement and discursive approach with fewer experts rather than to have a large sample of experts with less engagement with the guideline development process.

The finalised guideline will be distributed to all the participants in the study and to the departments of Health, Education and Social Development as well as to the relevant NGOs working on FASDs. The guideline will also be published in a peer-reviewed journal to add to the literature on FASDs.

\section{ETHICS AND DISSEMINATION}

Before the interviews and the focus group discussions (FGDs), aims and objectives of the study will be explained to the potential participants and they will be provided with an information sheet written in English explaining their roles. The potential participants will be requested to sign a consent form if they agreed to participate in the study. All participants for the interviews and FGDs will be asked to sign a consent form. All information obtained during the study will be kept strictly confidential in a computer with a password known only to the researchers in this study.

Acknowledgements We would like to thank Dorothy Badry, Carolyn Blackburn, Hayley Passmore and Roozen for reviewing this article.

Contributors BOA and AMB conceived and conceptualised the study and the paper. BOA designed and wrote the first draft of the manuscript. FCM redesigned the manuscript, contributed to the development of the paper and provided comments for improvement. KJO contributed to the development of the paper and provided comments for improvement. All authors read and approved the final manuscript.

Funding This research received no specific grant from any funding agency in the public, commercial or not-for-profit sectors.

Competing interests None declared.

Patient consent Not required.

Ethics approval The approval for the study was obtained from the research ethic $\mathrm{S}$ committee of the University of the Western Cape (BM/16/4/4), and further approvals were obtained from the Western Cape Department of Education (20161212 6937), Department of Health (WC_2016RP29_862) and Social Development (12/1/2/4).

Provenance and peer review Not commissioned; externally peer reviewed.

Open Access This is an Open Access article distributed in accordance with the Creative Commons Attribution Non Commercial (CC BY-NC 4.0) license, which permits others to distribute, remix, adapt, build upon this work non-commercially, and license their derivative works on different terms, provided the original work is properly cited and the use is non-commercial. See: http://creativecommons.org/ licenses/by-nc/4.0/

(c) Article author(s) (or their employer(s) unless otherwise stated in the text of the article) 2018. All rights reserved. No commercial use is permitted unless otherwise expressly granted.

\section{REFERENCES}

1. Chudley AE, Conry J, Cook JL, et al. Fetal alcohol spectrum disorder: Canadian guidelines for diagnosis. CMAJ 2005;172:S1-S21.

2. Jones KL, Smith DW. Recognition of the fetal alcohol syndrome in early infancy. Lancet 1973;302:999-1001.

3. Jones KL, Smith DW, Ulleland CN, et al. Pattern of malformation in offspring of chronic alcoholic mothers. Lancet 1973;1:1267-71.

4. Warren KR. A review of the history of attitudes toward drinking in pregnancy. Alcohol Clin Exp Res 2015;39:1110-7.

5. Streissguth AP, Barr HM, Kogan J, et al. Understanding the occurrence of secondary disabilities in clients with fetal alcohol syndrome (FAS) and fetal alcohol effects (FAE). Centers for Disease Control and Prevention 1996:96-6.

6. Riley EP, McGee CL. Fetal alcohol spectrum disorders: an overview with emphasis on changes in brain and behavior. Exp Biol Med 2005;230:357-65.

7. Riley EP, Infante MA, Warren KR. Fetal alcohol spectrum disorders: an overview. Neuropsychol Rev 2011;21:73-80.

8. Clark E, Lutke J, Minnes P, et al. Secondary disabilities among adults with fetal alcohol spectrum disorder in British Columbia. J FAS Int 2004;2:1-12.

9. Esper LH, Furtado EF. Identifying maternal risk factors associated with fetal alcohol spectrum disorders: a systematic review. Eur Child Adolesc Psychiatry 2014;23:877-89.

10. Olivier L, Curfs LMG, Viljoen DL. Fetal alcohol spectrum disorders: prevalence rates in South Africa. S Afr Med J 2016;106:103-6.

11. Urban M, Chersich MF, Fourie LA, et al. Fetal alcohol syndrome among grade 1 schoolchildren in Northern Cape Province: prevalence and risk factors. S Afr Med J 2008;98:877-82.

12. Urban MF, Olivier L, Viljoen D, et al. Prevalence of fetal alcohol syndrome in a South African city with a predominantly Black African population. Alcohol Clin Exp Res 2015;39:1016-26. 
13. May PA, Gossage JP, Marais AS, et al. The epidemiology of fetal alcohol syndrome and partial FAS in a South African community. Drug Alcohol Depend 2007;88:259-71.

14. May PA, Blankenship J, Marais AS, et al. Approaching the prevalence of the full spectrum of fetal alcohol spectrum disorders in a South African population-based study. Alcohol Clin Exp Res 2013;37:818-30.

15. May PA, de Vries MM, Marais AS, et al. The continuum of fetal alcohol spectrum disorders in four rural communities in South Africa: prevalence and characteristics. Drug Alcohol Depend 2016;159:207-18.

16. Jones KL, Streissguth AP. Fetal alcohol syndrome and fetal alcohol spectrum disorders: a brief history. J Psychiatry Law 2010;38:373-82.

17. Peadon $\mathrm{E}$, Rhys-Jones B, Bower $\mathrm{C}$, et al. Systematic review of interventions for children with fetal alcohol spectrum disorders. BMC Pediatr 2009;9:35-43.

18. Reid N, Dawe S, Shelton D, et al. Systematic review of fetal alcohol spectrum disorder interventions across the life span. Alcohol Clin Exp Res 2015;39:2283-95.

19. Rehm J, Rehn N, Room R, et al. The global distribution of average volume of alcohol consumption and patterns of drinking. Eur Addict Res 2003;9:147-56.

20. Kalichman SC, Simbayi LC, Cain D, et al. Alcohol expectancies and risky drinking among men and women at high-risk for HIV infection in Cape Town South Africa. Addict Behav 2007;32:2304-10.

21. Kalichman SC, Simbayi LC, Vermaak R, et al. HIV/AIDS risks among men and women who drink at informal alcohol serving establishments (Shebeens) in Cape Town, South Africa. Prev Sci 2008;9:55-62.

22. Kalichman SC, Simbayi LC, Vermaak R, et al. Randomized trial of a community-based alcohol-related HIV risk-reduction intervention for men and women in Cape Town South Africa. Ann Behav Med 2008;36:270-9.

23. Suliman S, Seedat S, Williams DR, et al. Predictors of transitions across stages of alcohol use and alcohol-use disorders in South Africa. J Stud Alcohol Drugs 2010;71:695-703.

24. Viljoen DL, Gossage JP, Brooke L, et al. Fetal alcohol syndrome epidemiology in a South African community: a second study of a very high prevalence area. J Stud Alcohol 2005;66:593-604.

25. Popova S, Chambers $C$. Fetal alcohol spectrum disorders must be recognized globally as a large public health problem. Int $\mathrm{J}$ Alcohol Drug Res 2014;3:1-3.

26. Cook JL, Green CR, Lilley CM, et al. Fetal alcohol spectrum disorder a guideline for diagnosis across the lifespan. CMAJ 2016;188:191-7.

27. Edition F. Diagnostic and statistical manual of mental disorders. 2013 https://psychiatryonline.org/pb-assets/dsm/update/DSM5Update October2017.pdf (accessed 28 Feb 2018).

28. National Organisation for Fetal Alcohol Spectrum Disorders. National FASD action plan. 2014 http://www.nofasd.org.au/policy-andadvocacy/national-fasd-action-plan (accessed 8 Jan 2018).

29. Fitzpatrick JP, Latimer J, Carter M, et al. Prevalence of fetal alcohol syndrome in a population-based sample of children living in remote Australia: the Lililwan Project. J Paediatr Child Health 2015;51:450-7.

30. Fitzpatrick JP, Latimer J, Olson HC, et al. Prevalence and profile of neurodevelopment and fetal alcohol spectrum disorder (FASD) amongst Australian Aboriginal children living in remote communities. Res Dev Disabil 2017;65:114-26.

31. Adnams CM. Fetal alcohol spectrum disorder in Africa. Curr Opin Psychiatry 2017;30:108-12.

32. Rendall-Mkosi K, London L, Adnams C, et al. Fetal Alcohol Spectrum Disorder in South Africa: situational and gap analysis: UNICEF, Pretoria, 2008. https://www.unicef.org/southafrica/SAF_resources fetalalcohol.pdf. (accessed 14 Sep 2017).

33. FASDSA - The Task Team. http://www.fasdsa.org/team.html (accessed 10 June 2017).

34. Marais S, Jordaan E, Olivier L, et al. Fetal alcohol spectrum disorders. In: Van Niekerk A, Suffla S, Seedat M, eds. Crime, violence and injury in South Africa: 21st century solutions for child safety:214-28.

35. Blas E, Koller T, Magar V, et al. WHO library cataloguing-inpublication data WHO Handbook for Guideline Development. Peer Rev Evidence-based Med World Heal Organ 2014;1:179.
36. World Health Organisation. WHO Handbook for Guideline Development: World Health Organisation, 2012. http://apps. who.int/ iris/bitstream/10665/75146/1/9789241548441_eng.pdf. (accessed 1 Mar 2018).

37. National Institute for Health and Care Excellence. Developing NICE guidelines: the manual. 2014 https://www.nice.org.uk/media/default/ about/what-we-do/our-programmes/developing-nice-guidelines-themanual.pdf (accessed 10 Jun 2017).

38. Information Technology Services Home SLU. Policies, standards, guidelines, procedures/processes: Saint Louis University Information Technology Services: SLU, 2017. http://www.slu.edu/its/policies. (accessed 10 Jun 2017).

39. Methley AM, Campbell S, Chew-Graham C, et al. PICO, PICOS and SPIDER: a comparison study of specificity and sensitivity in three search tools for qualitative systematic reviews. BMC Health Serv Res 2014; $14: 579$

40. Boland A, Cherry G, Dickson R. Doing a systematic review: a student's guide: Sage. https://books.google.co.za/books?hl=en

41. Petticrew M, Roberts $\mathrm{H}$. How to appraise the studies: an introduction to assessing study quality. Systematic reviews in the social sciences. Oxford: UK Blackwell Publishing Ltd:125-63.

42. Patton M. Qualitative evaluation and research methods: Sage, 1990. http://www.citeulike.org/group/108/article/107067. (accessed 1 Mar 2018).

43. Miles M, Huberman A. Qualitative data analysis: an expanded sourcebook: Sage, 1994. https://books.google.co.za/books?hl =en\&Ir=\&id=U4IU_-wJ5QEC\&oi=fnd\&pg=PR12\&dq=Qualitative+ data+analysis:+An+expanded+sourcecbook.\&ots=kETI6LOSUR\& sig=myBC5ImkUmJHO1z2hizEOBwQzD4. (accessed 1 March 2018).

44. Burnard $P$, Gill $P$, Stewart K, et al. Analysing and presenting qualitative data. Br Dent J 2008;204:429-32.

45. Creswell JW, Poth CN. Qualitative inquiry and research design: choosing among five approaches. SAGE 2016.

46. De Vos AS, Delport C, Fouche C, et al. Research at grass roots : for the social sciences and human services professions. Van Schaik 2011.

47. Tong A, Sainsbury P, Craig J. Consolidated criteria for reporting qualitative research (COREQ): a 32-item checklist for interviews and focus groups. Int J Qual Health Care 2007;19:349-57.

48. Govender B. The development of guidelines for social workers involved in early childhood development within the Department of Social Development. 2016 http://etd.uwc.ac.za/handle/11394/4972 (accessed 24 Aug 2017).

49. Pharaoh H, Frantz J, Smith M. Development, implementation and evaluation of youth development programmes to address health risk behaviour among grade 8 to grade 10 learners in selected schools in the Paarl area. 2014 http://etd.uwc.ac.za/bitstream/handle/11394/ 4297/pharaoh_h_phd_chs_2014.pdf?sequence=1\&isAllowed=y (accessed 24 Aug 2017).

50. Guidelines in designing a warm up program for the prevention of playing related musculoskeletal disorder among instrumentalists Adedayo Tunde Ajidahun 3102086. 2011; http://etd.uwc.ac.za/ bitstream/handle/11394/3035/Ajidahun_MSC_2011.pdf?sequence= 1\&isAllowed=y.

51. Rowe G, Wright G. The Delphi technique as a forecasting tool: issues and analysis. Int J Forecast 1999;15:353-75.

52. Hsu C-C. The Delphi technique: making sense of consensus. Pract assessment Res Eval 2007;12:1-8.

53. Keeney S, Hasson F, McKenna H. Consulting the oracle: ten lessons from using the Delphi technique in nursing research. J Adv Nurs 2006;53:205-12

54. Linstone H, Turoff M. The Delphi method. $1975 \mathrm{https} / / /$ pdfs.seman ticscholar.org/8634/72a67f5bdc67e4782306efd883fca23e3a3d.pdf (accessed 1 Mar 2018).

55. Watkins RE, Elliott EJ, Halliday J, et al. A modified Delphi study of screening for fetal alcohol spectrum disorders in Australia. BMC Pediatr 2013;13:13.

56. Donohoe $\mathrm{H}$, Stellefson M, Tennant B. Advantages and limitations of the e-Delphi technique. Am J Health Educ 2012;43:38-46.

57. Keeney S, Hasson F, McKenna HP. A critical review of the Delphi technique as a research methodology for nursing. Int J Nurs Stud 2001;38:195-200. 